Conclusions: The use of advanced techniques of ventilation assistance, associated with pre-surgical clinical stabilization and surgery performed in the NICU, during HFOV, have improved the outcome and the $\mathrm{CDH}$ neonatal survival.

1339

\section{RISK FACTORS FOR MECONIUM ASPIRATIONEM}

N. Raspopovic, T. Veljkovic

Neonatology, University of Belgrade, Belgrade, Serbia

The objective of this study was to identify link between fetal distress and respiratory distress (RD) in association with meconium stained liquor.In our hospital -a tertiary referral maternity hospital was born 6783 live newborns for one year during our prospective study.All infants born after meconim stained liquor who developed RD has:

1. fetal distress, proven with CTG, first $\mathrm{pH}$ after admission, Apgar score, asphyxia related complications (also were analysed mode of the delivery, sex, gestational age and birth body waight)

\section{2. maternal risk factors: fetal infection}

3. meconium aspirationem syndrome (MAS),PPHN and infection. $12 \%$ of newborns born alive for one year had meconium stained liquor, 74 term infants and 27 preterm developted RD. There were 58 term infants who had evidence of fetal distress and $67 \%$ had chorioamnionitis, 29\% MAS, 54\% develop PPHN. In preterm infants $87 \%$ had suspected infection. We conclude that asphyxia and infection are common in newborns who develop RD after meconim stained liquor.

\section{0}

\section{THE RESPIRATORY DISTRESS AS A CAUSE FOR ADMISSION TO THE PICU}

D. Tzira, K. Papazoglou, G. Sideri, A. Patsoura, A. Prezerakou, F. Petropoulou, J. Papadatos

PICU, Children's Hospital, Athens, Greece

Objective: The respiratory distress as a cause for admission in PICU

Material and methods: Studies have been retrospectively reviewed to children that had been hospitalized in the PICU "Aglaia Kyriakou" children's hospital from January 2006 until December 2009. From 702 admissions in period of 4 years, to 182 children (21\%) whose admission cause was respiratory distress. The patients were divided in seven categories:1)Viral infections of the respiratory tract-Bronciolitis 2)Bacterial infections of the respiratory tract, complicated or not 3) Cardiac disease 4)Malignant disease 5) Chronic disease and syndromes 6) Post operational respiratory failure 7) and other causes (i.e. intoxication).

Results: Viral infections-Bronchiolitis 60 children(33\%)-RSV(31\%), Bacterial pneumonia $29(16 \%)$ : complicated $14(7,7 \%)$ of known factor (staphaureus 50\%)and simple pneumonia 15(8,3\%). Cardiac disease 11 children had $(6 \%)$, malignante $13(7 \%)$, chronic sub causal disease(brain paralysis, neuromuscular disease or other syndromes) $34(19 \%)$. Postoperation respiratory failure 16 (9\%) other diseases 19 (10\%) as: ADEM(2), sdr Guillain Barre (3), acute obstruction of the airway (11), diaphragmatocele (1) , post traumatical contusion of the lungs (1), rabdomyolisis(1).

Therapy: Oxygen therapy were given $82(45 \%)$ children, controlled mechanical ventilation $87(47,8 \%)$ with minimal duration of ten days and NIV 12(6,5\%) with minimal duration 6 days in PICU, while 3 children from these continued mechanical ventilation at home. Thoracic drainage was required by 30 children, tracheostomy 6 and thoracectomydecortications 9

Conclusion: Respiratory distress is a common cause for admission in the PICU. Is the main symptom for various and different diseases, not including only the respiratory tract.

\section{1}

POSITIVE EXPIRATORY PRESSURE (PEP) FOR LOBAR COLLAPSE IN INFANTS

\author{
C. Ronan ${ }^{1}$, C. $\mathrm{Rae}^{2}, \mathrm{M}$. Vaidya ${ }^{3}$ \\ ${ }^{1}$ Paediatric Respiratory Physiotherapy, ${ }^{2}$ Barts \\ and the London Children's Hospital, ${ }^{3}$ Paediatric \\ Critical Care Unit, Barts and the London Children's \\ Hospital, London, UK
}

Background and aims: PEP is a commonly applied modality of chest physiotherapy in infants with chronic respiratory conditions such as cystic fibrosis. We report two cases of infants with acute right upper lobe collapse treated with PEP. 
Patient 1: A two month old baby was admitted with pertussis chest infection and was invasively ventilated for nine days and on CPAP for seventeen days. The baby had right upper-lobe collapse with ongoing oxygen requirement on day 31 of illness, having not resolved with conventional chest physiotherapy. PEP was initiated in supported sitting using an infant face mask and a $1.5 \mathrm{~mm}$ resistor. He received a total of 3 treatments over 2 days and was discharged home with no oxygen requirement and resolution of $\mathrm{X}$-ray changes prior to discharge.

Patient 2: Four week old ex-premature baby (31 weeks) was admitted with RSV positive bronchiolitis, ventilated for six days and on CPAP for five days. Radiographic findings showed a right upper lobe collapse, persisting despite regular conventional chest physiotherapy. PEP was started on day 12 using an infant face mask and a $1.5 \mathrm{~mm}$ resistor. On day 14 , a repeat chest $\mathrm{x}$-ray showed complete resolution of the right upper lobe collapse and the patient was discharged home the next day.

Conclusions: The use of PEP in infants is an under-reported but potentially beneficial modality in resolution of lobar collapse during acute illness.

\section{2}

\section{RISK FACTORS ASSOCIATED WITH EXTUBATION FAILURE (EF) IN EXTREMELY LOW GESTATIONAL AGE NEWBORNS (ELGAN)}

A.M. Sánchez Torres ${ }^{1}$, M.D. Elorza Fernández ${ }^{1}$, J. Pérez Rodríguez ${ }^{1}$, A. Díaz Redondo², J. Quero Jiménez ${ }^{1}$

\section{${ }^{1}$ Neonatology, ${ }^{2}$ Statistics, La Paz University} Hospital, Madrid, Spain

Aims: To analyze influence of perinatal characteristics(PC), mechanical ventilator(MV) parameters and early morbidity on EF of ELGAN with birth weight $(B W)<1000 \mathrm{~g}$.

Methods: This is a retrospective cohort of all ELGAN < 28 weeks gestational age(GA) and BW< $1000 \mathrm{~g}$ born between 2004-2009 with MV started in the first week of life. EF was defined as the need for reintubation within $48 \mathrm{~h}$ after the first extubation attempt.

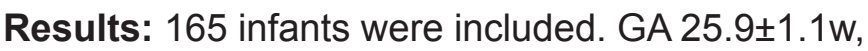
BW 788.2 $\pm 126.5 \mathrm{~g}$. First extubation attempt was at $11.5 \pm 14.6 \mathrm{~d}$ (median 6d). Nasal CPAP was used in all patients and nasal IMV in $78.4 \%$. EF was $30.9 \%$.
Comparing EF infants with those who succeed we didn't find any differences regarding PC. MV parameters and use of nasal IMV were similar between groups. EF group presented higher rates of late bronchopneumonia and MV length.

Extubation from A/C mode vs SIMV was earlier (7.6 vs $13.3 \mathrm{~d}, p=0.007$ ), with shorter time constant (51 vs 74.1, $p=0.000$ ) and higher mean airway pressure and rescue rate ( 7.1 vs $5.5 \mathrm{cmH}_{2} \mathrm{O}, \mathrm{p}=0.000 ; 39.4$ vs $24.5 \mathrm{rpm}, \mathrm{p}=0.000$ ); compliance, minute-volume and EF (29 vs $29.6 \%)$ were similar.

ELGAN with $B W<750 \mathrm{~g}$ had no significant difference in EF (37.3 vs $27.4 \%)$ although reintubation rate was higher (81.4 vs $51.4 \%, p=0.000)$.

Conclusion: This study does not support GA or $\mathrm{BW}$ as risk factors for $\mathrm{EF}$ in this population. BW< $750 \mathrm{~g}$ did not determine a higher EF rate, although it could be a risk factor for later reintubation.

Extubation from $\mathrm{A} / \mathrm{C}$ mode allows early extubation without increase of EF.

\section{3}

\section{UNSAFE SWALLOW IN CHILDREN: ARE WE DOING ENOUGH?}

\author{
N. Kumaraguru, M. Vaidya, S. Thyaga Rajan
}

Paediatric Critical Care Unit, Barts and the London Children's Hospital, London, UK

Background: Recurrent chest infections are a common problem in children with unsafe swallow. Tracheostomy and gastrostomy are commonly performed in such children. However, it is not clear whether these interventions will resolve the problem. Aim: Report the clinical profile of patients with unsafe swallow who have undergone tracheostomy and gastrostomy.

Methods: We retrospectively analysed children admitted to Paediatric Critical Care Unit in a tertiary referral hospital (March 2009-March 2010). We reviewed the indications for tracheostomy, associated co-morbidities, feeding adjuncts used, safety of swallow and the incidence of recurrent chest infections.

Results: Ten children were managed during this period with a median age at tracheostomy was 8 months (Range 1 week to 15 years). Common indications for tracheostomy included failed / difficult intubation (2), bilateral vocal cord paralysis 